\title{
CALIDAD DE VISIÓN Y SATISFACCIÓN DEL PACIENTE USUARIO DE PANTALLAS DE VISUALIZACIÓN DE DATOS TRAS CIRUGÍA REFRACTIVA
}

\author{
Ma ISABEL RUBIO CUEVAS(*), JORGE L. ALIÓ SANZ(**) Y JOSÉ R. LOBATO CAÑÓN(***)
}

(*) Médico del trabajo. Servicio de prevención, Universidad de Valencia.

(**) Catedrático de oftalmología. Facultad de Medicina. Universidad Miguel Hernández de Elche (Alicante). (***) Escuela Profesional de Medicina del Trabajo. Universidad Miguel Hernández de Elche (Alicante).

\section{RESUMEN}

OBJETIVO: Evaluar la influencia en la calidad de vida y satisfacción de los trabajadores usuarios de pantallas de visualización de datos (PVD) que se asocia a la mejora de la función visual tras queratomileusis in situ con láser excimer (LASIK).

MÉTODOS: Estudio observacional y longitudinal en una población de 96 pacientes intervenidos de cirugía refractiva con láser excimer según técnica LASIK mediante aplicación de un cuestionario construído al efecto aplicado antes y después de cirugía por un observador independiente.

RESULTADOS: Agudeza visual y refracción ocular han mejorado tras LASIK, siendo el índice de eficacia de 1.1.

La sensibilidad al contraste tras cirugía mejora en todas las frecuencias $(6 \mathrm{c} / \mathrm{g}, 3 \mathrm{c} / \mathrm{g}$ y $1 \mathrm{c} / \mathrm{g})$ en el ojo derecho. Mientras que en el ojo izquierdo no hay diferencias significativas.

La calidad de visión con y sin corrección es mejor tras LASIK (significativa $\mathrm{p}<0.05$ ). Volverían a intervenirse y recomendarían este procedimiento quirúrgico a otros pacientes el $84.38 \%$ de pacientes.

CONCLUSIONES: Los resultados muestran que el LASIK mejora la función visual y la calidad de vida de estos pacientes.

PALABRAS CLAVE: cirugía refractiva, calidad de vida, función visual, calidad de visión, pantallas de visualización de datos.

\section{SUMMARY}

PURPOSE: To evaluate the influence in quality of vision and satisfaction of video display terminal (VDT) users related to improvement of visual functions following excimer laser in situ keratomileusis (LASIK).

METHODS: Longitudinal and observational study in a population of 96 patients that underwent LASIK using a questionnaire made for that, applied before and after surgery by an independent observer.

RESULTS: Visual acuity and ocular refraction have improved after LASIK, being the efficiency index 1.1 .

Contrast sensitivity postoperative improved at all spatial frequencies in the right eye $(6 \mathrm{c} / \mathrm{g}, 3 \mathrm{c} / \mathrm{g}$ and $1 \mathrm{c} /$ ). While in the left eye there were no significant differences.

Quality of vision with and without correction is also better after LASIK $(p<0.05)$. And $84.38 \%$ of patients would repeat themselves and recommend this surgery to other patients.

CONCLUSIONS: The results show that LASIK improves visual function and quality of life of patients.

KEY WORDS: refractive surgery, quality of life, visual function, quality of vision, video display terminals. 


\section{INTRODUCCIÓN}

Los defectos de refracción del globo ocular miopía, hipermetropía y astigmatismo requieren corrección óptica para conseguir una agudeza visual normal. Clásicamente las gafas y lentes de contacto han corregido estos defectos pero en las últimas décadas se ha desarrollado la cirugía refractiva en un intento de mejorar la calidad de vida de los pacientes ${ }^{1}$.

En concreto la cirugía refractiva con láser según la técnica LASIK (láser in situ queratomileusis) consiste en el esculpido óptico de la córnea mediante la aplicación del láser en el espesor del tejido (ablación intraestromal) en lugar de la superficie externa. Para ello se levantan las primeras láminas del tejido corneal, algo más de una décima de milímetro de espesor mediante el microqueratomo. El láser actuará sobre el lecho descubierto al levantar dichas capas (el flap corneal). La curvatura y por tanto el poder óptico cambian en función de la cantidad de tejido que el láser volatiliza.

Esta es una cirugía electiva demandada por un nicho de población creciente para eliminar una disfunción visual que afecta a la calidad de vida del individuo.

La cirugía refractiva proporciona unas óptimas condiciones visuales de forma que una mejor eficiencia visual puede originar: incremento de la productividad, menor número de accidentes y por consiguiente disminución de los costes de los seguros y descenso del absentismo ya que la tarea es menos fatigante según una consideración visual. Además aumenta las posibilidades laborales, la satisfacción en el trabajo y el rendimiento, y además facilita la integración en la vida social y laboral.

En este estudio nos planteamos los siguientes objetivos:

Evaluar la influencia de la cirugía refractiva sobre la calidad de visión, calidad percibida de visión y grado de satisfacción percibida por el paciente usuario de pantallas de visualización de datos.

Analizar la influencia de los cambios en la calidad percibida de visión del paciente usuario de pantallas de visualización de datos, cumplimiento de las expectativas previas a la cirugía refractiva y comparación de los resultados con las pruebas oftalmológicas y optométricas (agudeza visual con y sin corrección, refracción ocular, equilibrio muscular, pupilometría y prueba de sensibilidad al contraste) antes y después de cirugía refractiva.

\section{MATERIAL Y MÉTODOS}

Estudio observacional y longitudinal en una población de 96 pacientes intervenidos con cirugía refractiva con láser excimer según técnica LASIK, mediante aplicación de un cuestionario construido al efecto aplicado antes y después de cirugía por un observador independiente.

El cuestionario está basado en el test VF- $14^{2}$ y otros test ${ }^{3,4}$ validados.

Se ha aplicado la normativa descrita por D. $\mathrm{Koch}^{5}$ comparando la eficacia del procedimiento quirúrgico empleado.

Los sujetos de estudio fueron los pacientes intervenidos, siguiendo el mismo protocolo quirúrgico, en la Unidad Clínica de Cirugía Refractiva de VISSUM Alicante entre enero de 2003 y enero de 2005. Las edades de los pacientes estaban comprendidas entre los 21 y los 60 años. Entre ellos se encuentran 54 mujeres y 42 hombres.

Estos pacientes fueron intervenidos de los siguientes defectos refractivos: miopía desde -1 a 10 dioptrías, hipermetropía desde +3 dioptrías hasta + 6 dioptrías, astigmatismos mayor o igual a 2,5 dioptrías hipermétropes y astigmatismos mayor o igual a 2,5 dioptrías miópicos.

Los pacientes se distribuyen en:

- grupo 1: miopes desde -1 a - 10 dioptrías

- grupo 2: hipermétropes desde +3 dioptrías hasta + 6 dioptrías

- grupo 3: astígmatas con astigmatismo mayor o igual a 2,5 dioptrías hipermétropes y miópicos.

En cada grupo hay 32 pacientes.

Estos pacientes para ser seleccionados tenían que ser usuarios de pantallas de visualización de datos en su trabajo habitual. Se ha considerado en este estudio trabajador usuario de pantallas de visualización de datos tanto el que las utiliza más de 4 horas/día como el que menos de 2 horas/día.

El material utilizado fue el siguiente:

- Agudeza visual: optotipos de Snellen, proyectados en un monitor de televisión con el sistema Mentor. Ohio. USA. 
- Refracción: caja de lentes estándar, retinoscopio de punto. Welch-Allyn. USA.

- Diámetro pupilar: pupilómetro. Colvard pupillometer. Oasis. Glendova, California, USA.

- Quirófano con láser excimer Chiron Tecnolas 217-C ( Bausch \& Lomb Surgical).

Los resultados de las mediciones realizadas con este material a cada paciente antes y después de cirugía refractiva, se extraen de la historia clínica correspondiente.

- Prueba de sensibilidad al contraste: CVS 1000E, Vector Vision, Dayton, Ohio, USA.

Esta prueba se realiza por un observador independiente antes y después de cirugía a todos los pacientes, que fueron examinados utilizando su mejor corrección óptica.

En cada examen se analiza la sensibilidad al contraste para frecuencias altas (6 ciclos/ grado), medias (3 ciclos/grado) y bajas (1 ciclo/grado). Esta prueba determina la calidad de visión obtenida con el tratamiento lasik de forma superior al optotipo de Snellen.

Las variables que aparecen en el cuestionario miden la calidad de visión y la satisfacción del paciente.

Calidad de visión: Medida de los conceptos calidad percibida de visión y calidad de visión mediante diferentes indicadores:

- Dimensión física: conjunto de variables clínicas y objetivas que determinan el estado de visión del paciente, independientemente de las necesidades y exigencias visuales que le generan sus actividades laborales y cotidianas.

Se realiza la medida clínica de: refracción visual, agudeza visual, sensibilidad al contraste, equilibrio oculomotor y diámetro pupilar.

- Dimensión psicológica: medir la percepción subjetiva del individuo acerca de su calidad de visión, valoración sobre su calidad de visión percibida general y consideraciones respecto a cirugía refractiva y calidad de vida y calidad de visión antes y después de cirugía refractiva. Y sobre su visión nocturna.

El cuestionario y las pruebas oftalmológicas fueron realizados en dos fases: la $1^{\mathrm{a}}$ unas horas previas a la cirugía refractiva y la $2^{\mathrm{a}}$ entre 1 y 6 meses poste- riores a la cirugía refractiva. Todos los resultados obtenidos fueron evaluados estadísticamente para comprobar su grado de significación con la ayuda del programa informático SPSS versión 12.0.

\section{RESULTADOS}

El índice de eficacia según el método descrito por D. Koch es de 1.1, por tanto el procedimiento quirúrgico es eficaz, los pacientes han ganado visión.

La refracción también ha mejorado ya que tras cirugía la mayoría necesitan llevar menor corrección.

La sensibilidad al contraste tras cirugía mejora en todas las frecuencias estudiadas (6 ciclos/grado, 3 ciclos/grado y 1 ciclo/grado) en el ojo derecho, existiendo diferencias estadísticamente significativas mientras que en el ojo izquierdo no ha cambiado tras cirugía para todas las frecuencias.

En cuanto al equilibrio oculomotor y la pupilometría no existen diferencias significativas antes y después de la cirugía estando ambos parámetros dentro de la normalidad en la mayoría de pacientes.

El motivo principal para operarse fue la mejora de la visión en el $62.5 \%$ de trabajadores seguido de depender menos de las gafas en el $41.7 \%$.

Se obtiene que la calidad de visión con corrección es mejor tras LASIK (significativa $\mathrm{p}<0.05$ ). Figura 1. La calidad de visión sin corrección también es mejor tras LASIK $(\mathrm{p}<0.05)$. Figura 2.

La mayoría refieren ver peor durante la noche y tener problemas visuales en la conducción nocturna.

Tras cirugía la mayoría refieren tener menores dificultades con las gafas y las lentillas siendo la diferencia estadísticamente significativa $(\mathrm{p}<0.05)$.

La mayoría de estos trabajadores no refieren visión doble, imágenes fantasma ni cambios en la visión durante el día, no existen diferencias significativas antes y después de la cirugía.

En cambio los problemas durante la lectura antes de cirugía ocurren en el $61.46 \%$ y tras cirugía en el $46.88 \%$ de pacientes, por tanto tras cirugía son menores $(p<0.05)$.

La distorsión de la visión de los detalles antes de cirugía ocurre en el $44.79 \%$ de pacientes y después en el $20.83 \%$, por tanto también es menor tras cirugía $(\mathrm{p}<0.05)$. 
Figura 1. Calidad de visión con corrección.

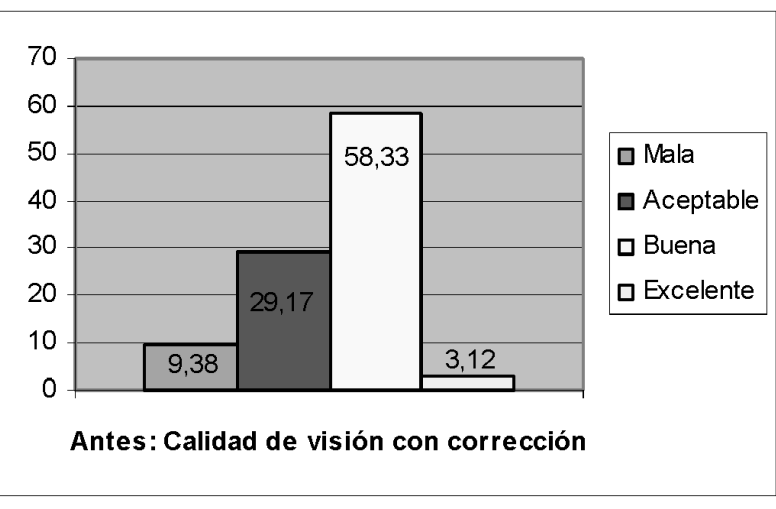

Antes de cirugía

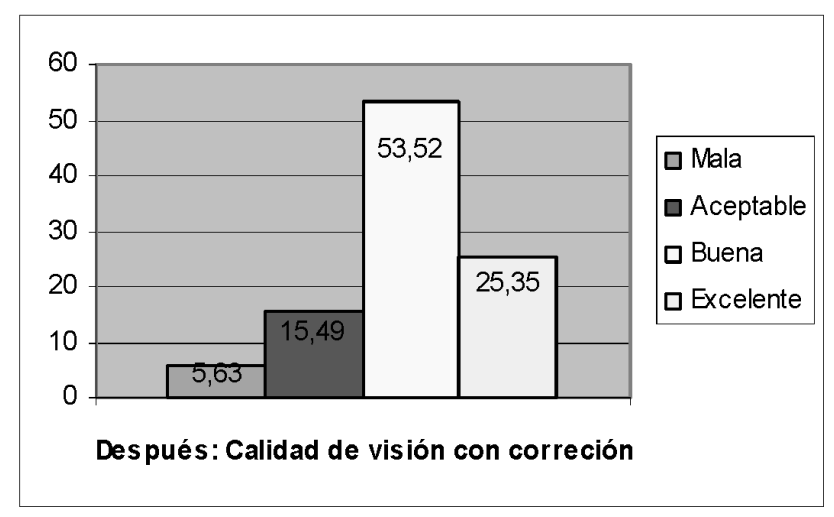

Después de cirugía

Figura 2. Calidad de visión sin corrección.

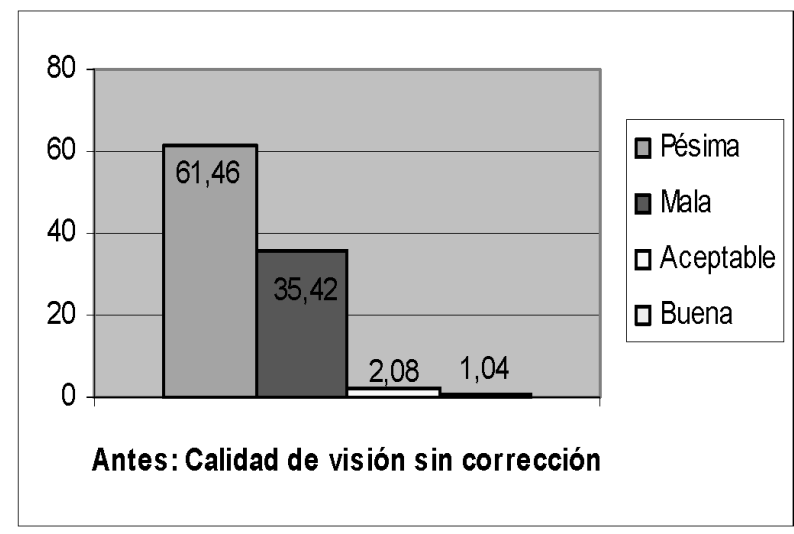

Antes de cirugía

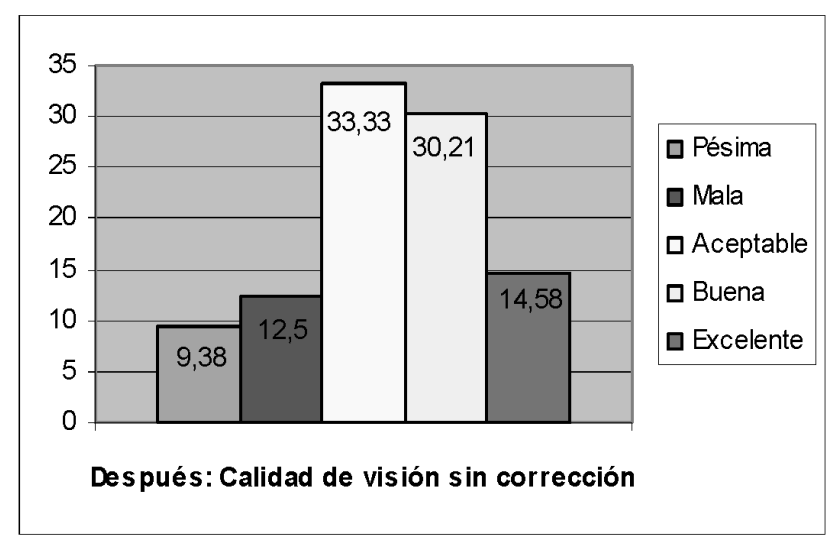

Después de cirugía
Es más habitual la existencia de halos nocturnos (75\% pacientes) y deslumbramiento $(68.75 \%$ pacientes) después de operarse.

Respecto a las expectativas visuales a obtener con la intervención podemos observarlas en la figura 3, la conveniencia se entiende por comodidad.

El $65.62 \%$ de pacientes consideran que su calidad percibida de visión ha mejorado, sobre todo los miopes.

La estabilidad de la visión tras cirugía podemos observarla en la figura 4.
En cuanto a las condiciones que han cambiado tras cirugía la principal es la mejora de la visión en el $82.29 \%$ de estos trabajadores seguido de una mayor participación en el trabajo en el $47.91 \%$ de ellos.

Están satisfechos con el resultado obtenido el $79.17 \%$ de pacientes, siendo este porcentaje mayor en los astígmatas donde hay un $81.3 \%$ de satisfechos. Volverían a intervenirse y recomendarían este procedimiento quirúrgico a otros pacientes el $84.38 \%$ de pacientes. 
Figura 3. Expectativas visuales a obtener con la intervención.

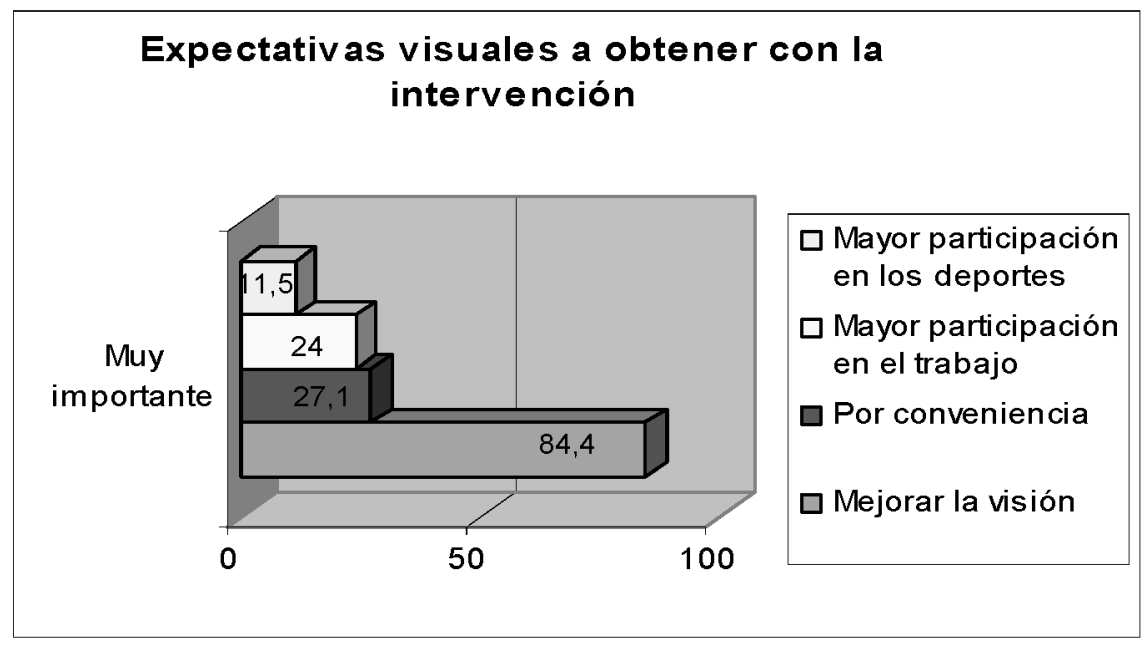

Figura 4. Visión estable tras cirugía.

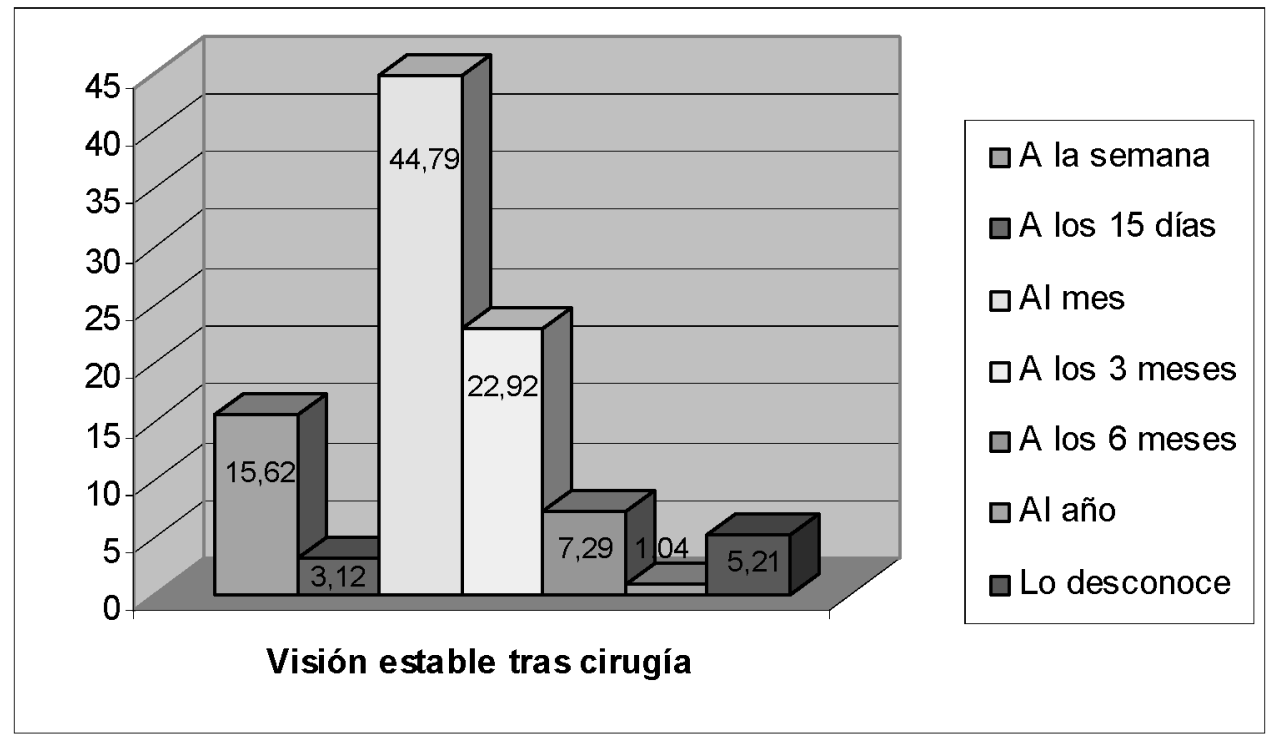

\section{DISCUSIÓN}

En cuanto a la eficacia del procedimiento quirúrgico según el método descrito por D. Koch, todos los individuos han ganado visión con este procedimiento (el resultado es estadísticamente significativo), lo que se traduce en que sin corrección tras la cirugía ven mejor que antes con corrección. Sobre todo astígmatas y miopes, como se ha visto en otros estudios ${ }^{6,7}$.
Respecto a la refracción ocular en el astigmatismo se observa una reducción de la refracción postoperatoria para todos los trabajadores, al igual que en el estudio de Albarrán-Diego ${ }^{8}$ et al. Esta reducción del astigmatismo puede mejorar la agudeza visual por la reducción del la distorsión de la imagen que supone, por eso la agudeza visual tras cirugía en el grupo de astígmatas de este estudio ha obtenido resultados tan buenos. 
En la miopía la refracción postoperatoria es prácticamente cero, la mayoría no necesitan refracción. Aunque en este caso la mejoría de la agudeza visual es sólo en el ojo derecho, esta diferencia puede deberse al estado dióptrico previo en el ojo izquierdo. Si éste tiene mayor nivel de dioptrías los resultados, es de esperar, que sean peores.

En la hipermetropía también hay un significativo descenso de la refracción postoperatoria. No obstante, como ya se ha visto en otros estudios ${ }^{9,10}$ la corrección de altos rangos de hipermetropía con LASIK es menos segura y predecible que para bajos rangos. Parece que el punto de corte está entre 4 y 5 D. Por tanto, puede que en nuestro estudio al tener mayores rangos de hipermetropía que los del punto de corte, existan peores resultados tanto de agudeza visual postoperatoria como de refracción.

En la prueba de sensibilidad al contraste obtenemos que en las diferentes frecuencias espaciales valoradas $(6,3$ y $1 \mathrm{cpg})$ los cambios entre el control preoperatorio y el postoperatorio son significativos para todas las frecuencias en el ojo derecho. Mientras que no se aprecia un cambio significativo en ninguna frecuencia para el ojo izquierdo. Luego se puede concluir que tras la cirugía, la sensibilidad al contraste mejora en todas las frecuencias en el ojo derecho, como se ha demostrado en otros estudios ${ }^{11,12}$.

El resultado obtenido con el ojo izquierdo requeriría un estudio más extenso, en que se analizara porqué el resultado es distinto al otro ojo. Por ejemplo correlacionando la sensibilidad al contraste con el equivalente esférico preoperatorio.

Estos trabajadores consideran su visión nocturna bastante peor que la diurna, al igual que en otros estu$\operatorname{dios}^{13}$ al respecto.

Tras la operación estos trabajadores sufren más deslumbramiento, como se ha descrito en otros trabajos $^{14}$, excepto en el grupo de miopes en el que no ha habido diferencias significativas antes que después de operarse. Esto puede deberse a que el deslumbramiento subjetivo que se produce tras LASIK alcanza un pico 1 mes después de la operación y luego disminuye ${ }^{15}$. En este grupo de pacientes el cuestionario post-cirugía se cumplimenta pasado ese primer mes, por tanto existe menos deslumbramiento.

Tras la operación estos trabajadores (el $75 \%$ ) refieren halos nocturnos. Este resultado es comparable a lo descrito en trabajos previos: halos nocturnos en $62 \%$ de pacientes hipermetrópicos 14 y el $28 \%$ de pacientes miopes ${ }^{16}$.
La motivación principal de los pacientes para esta cirugía fue la mejoría de su agudeza visual seguida de la eliminación de la dependencia de las gafas. Este resultado es comparable con estudios previos ${ }^{17}$.

Los hipermétropes y astígmatas esperaban obtener una mayor calidad percibida de visión con esta cirugía que la que luego han obtenido realmente. Mientras que los miopes sí han visto cumplidas sus expectativas de mejorar su calidad percibida de visión tras operarse. Luego los resultados en cuanto a calidad de visión percibida son excelentes en el grupo de miopes.

Hay que tener en cuenta que en este resultado también puede influir la severidad del defecto refractivo preoperatorio. Puesto que es un predictor significativo para la mejora de la calidad percibida de visión después del LASIK $^{18}$. Los pacientes con mayores defectos refractivos preoperatorios tienen menor mejora de la función visual después del LASIK.

La estabilidad subjetiva de la visión se produjo en la mayoría de pacientes al mes, resultado comparable a lo descrito en trabajos previos ${ }^{17,19}$.

La mayoría de trabajadores contestó estar satisfecho con el resultado de la intervención, resultado análogo al de estudios previos ${ }^{17,14,20}$ al respecto. El mayor porcentaje de satisfechos es el de astígmatas, aunque esta diferencia es difícil de evaluar porque existen muchas variables que influyen en la satisfacción con el resultado como ${ }^{21}$ : edad del paciente, tamaño de la zona de ablación, retratamientos, etc.

En todos los grupos hay alguna persona insatisfecha, esto puede deberse a molestias subjetivas secundarias al LASIK o a falsas expectativas sobre la mejoría de la calidad visual.

La mayoría, sobre todo los miopes también recomendarían la técnica a otras personas. Resultado obtenido en otros trabajos ${ }^{17,22,23}$. Hay que tener en cuenta que recomendar esta cirugía a otros no es una medida en sí misma válida de la satisfacción después del LASIK ${ }^{23}$.

\section{CONCLUSIONES}

La calidad de visión con y sin corrección percibida por estos trabajadores mejora tras cirugía refractiva así como las dificultades con las gafas o lentillas que son menores.

El equilibrio muscular y la pupilometría no se han visto influídos por la cirugía refractiva. Mientras que 
la agudeza visual y la refracción ocular han mejorado tras cirugía. El índice de eficacia es de 1,1.

Casi la totalidad de pacientes consideran el resultado obtenido como satisfactorio, manifestan- do su deseo de volver a ser intervenido si se reprodujeran las condiciones preoperatorias y recomendando este procedimiento quirúrgico a otros pacientes.

\section{BIBLIOGRAFÍA}

1. Fernández Vega L, Alió J, Barahona

$\mathrm{JM}$, et al. Informe sobre láser excimer en oftalmología. Arch. Soc. Esp. Oftalmol. 1995; 68: 315-324.

2. Steinberg EP, Tielsch JM, Schein OD, et al. The VF-14. An index of functional impairment in patients with cataract. Arch Ophthalmol 1994; 112: 630-638.

3. Gunter K, Seiler T, Wollensak J. Report on psychosocial findings and satisfaction among patients 1 year after excimer laser photorefractive keratectomy. Refractive \& Corneal Surgery 1992; volume 8: 286-289.

4. Brunette I, Gresset J, Boivin JF, et al. Functional outcome and satisfaction after photorefractive keratectomy. Ophthalmology 2000; 107: 1783-1796.

5. Koch DD, Kohnen T, Obstbaum SA, Rosen ES. Format for reporting refractive surgical data. J Cataract Refract Surg. 1998; 24: 285-7.

6. Nepomuceno RL, Boxer Wachler BS, Sato M, Scruggs R. Use of large optical zones with the LADARVision laser for myopia and myopic astigmatism. Ophthalmology. 2003; 110: 1384-1390.

7. Doughman DJ, Hardten DR. Loss of vision after laser in situ keratomileusis. Eye \& Contact lens. 2003; 29 (1 suppl): S135-8.

8. Albarrán-Diego, C; Muñoz, G; Montes-Micó, R; MPhil; Alió, JL. Bitoric laser in situ keratomileusis for astigmatism. J Cataract Refract Surg. 2004; 30: 14711478.

9. Cobo-Soriano R, Lloret F, González-López F, et al. Factors that influence outcomes of hyperopic laser in situ keratomileusis. J Cataract Refract Surg. 2002; 28: 1530-8.

10. Varley GA, Huang D, Rapuano CJ, et al. LASIK for hyperopia, hyperopic astigmatism and mixed astigmatism. Ophthalmology 2004; 111: 1604-1617.

11. Pérez Santonja, JJ; Sakala, HF; Alió, JL. Contrast sensitivity after laser in situ keratomileusis. J Cataract Refract Surg. 1998 Feb; 24 (2): 138-9.

12. Cardona Ausina, C; Pérez Santonja, JJ; Ayala Espinosa, MJ; et al. Contrast sensitivity after laser in situ keratomileusis for myopia ( LASIK-M). Arch Soc Esp Oftalmol 2000 Aug; 75 (8): 541-6.
13. Hill JC. An informal satisfaction survey of 200 patients after laser in situ keratomileusis. J Refract Surg. 2002; 18: 454-459.

14. Pérez Santonja JJ, Sakla HF, Ayala Espinosa MJ, Cardona Ausina C, Alió JL. Queratomileusis in situ con laser excimer (LASIK) para hipermetropía: resultados preliminares. Arch Soc Esp Oftalmol. 1999; 74: 89-96.

15. Lackner B, Pieh S, Schmidinger G, et al. Glare and halos phenomena after laser in situ keratomileusis. J Cataract Refract Surg. 2003; 29: 444-450.

16. Pérez Santonja JJ; Alió JL, Ismail MM, Sánchez Pego JL: Queratomileusis in situ con láser excimer: resultados preliminares. Arch Soc. Esp. Oftalmol. 1998; 73: 93-98.

17. Pazos González B, Sanz Piury M, Sánchez Salorio $\mathrm{M}$. Análisis de la motivación y de los niveles de satisfacción en la cirugía refractiva con láser excimer. Microcirugía ocular. 1998; $\mathrm{n}^{\circ} 2$.

18. Lee J, Lee J, Park K, et al. Assessing the value of laser in situ keratomileusis by patient reported outcomes using quality of life assessment. J Refract Surg 2005; 21: 59-71.

19. el-Agha MS, Johnston EW, Bowman RW, et al. Excimer laser treatment of spherical hyperopia: PRK or LASIK ? Trans Am Ophthalmol Soc 2000; 98: 59-66, discussion 66-9.

20. Hejcmanova M, Horaickova M, Vlkova E. Evaluation of visual function and changed quality of life among patients after excimer refractive surgery. Cesk Slov Oftalmol. 2005 Jan; 61 (1): 50-6.

21. Hammond, Stephen DJr; Puri, Anil K; Ambati, Balamurali K. Quality of vision and patient satisfaction after LASIK. Current opinion in Ophthalmology. 2004; 15 (4): 328-332.

22. Bailey MD, Mitchell GL, Dhaliwal DK, et al. Patient satisfaction and visual symptons after laser in situ keratomileusis. Ophthalmology. 2003; 110: 1371-1378.

23. Bailey MD, Mitchell GL, Dhaliwal DK, et al. Reasons patients recommend laser in situ keratomileusis. J Cataract Surg. 2004; 30: 1861-1866. 